\title{
Croissance radiale et réponse au climat du pistachier de l'Atlas (Pistacia atlantica Desf.) dans le Parc national de Theniet El Had (Algérie)
}

Naïma IFTICENE-HABANI ${ }^{1,2}$

Fatiha ABDoun ${ }^{3}$

${ }^{1}$ Institut national de recherche forestière Arboretum de Baïnem

BP 37, Chéraga

16014 Alger

Algérie

2 Université Alger 1.

Faculté des Sciences

Département Science de la nature et de la vie

02, Rue Didouche Mourad, 16000 Alger

Algérie

${ }^{3}$ Université des sciences

et de la technologie Houari Boumediene (USTHB)

Faculté des sciences biologiques

Laboratoire écologie et environnement BP 32, El Alia, Bab Ezzouar 16111 Alger

Algérie

Auteur correspondant /

Corresponding author:

Naima Ifticene-Habani -

naimaifticene@hotmail.com



Photo 1.

Vue du versant nord de Djebel Meddad où le pistachier de l'Atlas se mélange au cèdre de l'Atlas (avril 2012).

Photo F. Abdoun.

\section{- Ccirad (6)}

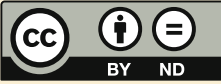

Licence Creative Commons :

Attribution - Pas de Modification 4.0 International.

Attribution-NoDerivatives 4.0 International (CC BY-ND 4.0)
Citer l'article / Cite the article

Ifticene-Habani N., Abdoun F., 2018. Croissance radiale et réponse au climat du pistachier de l'Atlas (Pistacia atlantica Desf.) dans le Parc national de Theniet El Had (Algérie). Bois et Forêts des Tropiques, 335 : 3-13. Doi : http://dx.doi.org/10.19182/bft2018.335.a31496 


\section{RÉSUMÉ}

Croissance radiale et réponse au climat du pistachier de l'Atlas (Pistacia atlantica Desf.) dans le Parc national de Theniet El Had (Algérie)

En Afrique du Nord, l'aire de répartition du pistachier de l'Atlas s'étend depuis l'Atlas tellien, où il se mêle au cèdre de l'Atlas, jusqu'au Sahara central où il côtoie l'acacia. Il possède une amplitude écologique remarquable et paraît candidat à une extension spatiale, à la faveur du réchauffement climatique. Après avoir rendu compte, dans un précédent article, des relations cerne-climat de cet arbre selon un gradient d'aridité, le présent travail concerne le déterminisme climatique de sa croissance radiale en milieu forestier, sous bioclimat subhumide. L'étude a été réalisée à Djebel Meddad sur deux expositions (nord et sud). L'analyse de la réponse aux événements climatiques extrêmes par le calcul des années caractéristiques révèle que le pistachier de l'Atlas est plus sensible aux événements extrêmes dans les expositions chaudes que fraîches, plus particulièrement aux événements qui inhibent la croissance. Les sensitivités moyennes sont très proches sur les deux versants mais restent faibles par rapport à celles obtenues dans les régions arides et semi-arides. L'analyse des indices de croissance montre que tous les cernes minces ou épais correspondent à des conditions particulièrement sèches ou pluvieuses. Les températures moyennes annuelles n'ont qu'un effet complémentaire. Aucun cerne extrêmement mince n'est enregistré au niveau des deux versants. Les fonctions de réponse montrent que les facteurs climatiques de l'année en cours jouent un rôle majeur dans la construction du cerne.

Mots-clés : croissance radiale, pistachier de l'Atlas, relations cerne-climat, subhumide, Theniet El Had, Algérie.

\section{ABSTRACT}

\section{Radial growth and response to climate of the mount Atlas mastic tree (Pistacia atlantica Desf.) in Algeria's Theniet El Had National Park}

The distribution range of the Mount Atlas mastic tree in North Africa extends from the Tellian Atlas mountains, where it is found together with Atlas cedars, to the central Sahara where it grows with acacias. It has a very wide ecological range making it a suitable candidate for extension with the advance of climate change effects. To follow a previous article on the relationship between climate and growth rings, this paper reports on the way climate determines the tree's radial growth in forested environments in a sub-humid bioclimate. The study was conducted in two stands on the slopes of Djebel Meddad, one south-facing and one north-facing. Our analysis of responses to extreme climatic events based on calculations for characteristic years shows that the species is more sensitive to extremes in warmer conditions of exposure, especially when these events inhibit growth. Average sensitivities are very similar on both slopes but low compared to those obtained for arid and semi-arid regions. Analysis of growth indicators shows that thin and thick growth rings always correspond to especially dry and especially rainy conditions. Average annual temperatures only have an additional effect. No very thin growth rings were recorded on either slope. The response functions show that climatic factors in a given year play a major role in the construction of growth rings.

Keywords: radial growth, Mount Atlas mastic tree, climate-growth ring relationships, sub-humid, Theniet El Had, Algeria.

N. Ifticene-Habani, F. Abdoun

\section{RESUMEN}

\section{Crecimiento radial y respuesta al clima del almácigo del monte Atlas (Pistacia atlantica Desf.) en el Parque Nacional de Theniet El Had (Argelia)}

El área de distribución del almácigo en África del Norte se extiende desde el Atlas telliano, donde se mezcla con el cedro del Atlas, hasta el Sáhara central en donde coexiste con la acacia. Esta notable amplitud ecológica lo convierte en un posible candidato a la extensión espacial propiciada por el calentamiento climático. En un artículo anterior, informábamos de las relaciones anillos-clima de este árbol según un gradiente de aridez. El presente artículo aborda el determinismo climático de su crecimiento radial en un medio forestal de bioclima subhúmedo. El estudio se realizó en Djebel Meddad y en dos exposiciones (norte y sur). El análisis de respuesta a eventos climáticos extremos a través del cálculo de años característicos revela que el almácigo es más sensible a eventos extremos en las exposiciones cálidas que en las frescas y, sobre todo, a los eventos que inhiben el crecimiento. El promedio de sensibilidad es muy parecido en ambas vertientes y presenta un valor bajo comparado con los de regiones áridas y semiáridas. El análisis de los índices de crecimiento muestra que todos los anillos, finos o gruesos, corresponden a condiciones particularmente secas o húmedas. Las temperaturas medias anuales sólo tienen un efecto complementario. No se observaron anillos extremadamente finos en ninguna de las dos exposiciones. Las funciones de respuesta muestran que los factores climáticos del año en curso desempeñan un papel fundamental en la formación del anillo.

Palabras clave: crecimiento radial, almácigo del monte Atlas, relaciones anillo-clima, subhúmedo, Theniet El Had, Argelia. 


\section{Introduction}

Dans le bassin méditerranéen, les scénarios climatiques prédisent une diminution des précipitations pendant la saison de croissance végétale et une augmentation de la fréquence des événements extrêmes (IPCC, 2013). Ces changements devraient très probablement générer une aridité accrue dans une région déjà très contrainte par la sécheresse estivale, et entraîner ainsi une baisse de la survie et de la croissance des espèces (Sala et al., 2000).

L’Algérie a connu depuis plus de deux décennies un déficit pluviométrique et une persistance de la sécheresse, en particulier dans la partie occidentale et les hauts plateaux (Benabadji et Bouazza, 2000 ; Hirche et al., 2007 ; Hassini et al., 2015). Les différents symptômes observés suite aux canicules sur une grande variété d'essences et dans une très large gamme de conditions stationnelles ont rappelé d'une façon spectaculaire le rôle clé du climat dans la vitalité des écosystèmes (Landmann et al., 2003 ; Bréda et al., 2004).

Le pistachier de l'Atlas nous paraît être une essence capable de résister au réchauffement. Son adaptation aux milieux les plus variables (depuis l'Atlas tellien jusqu'à l'Ahaggar au Sahara central) le rend particulièrement intéressant pour les programmes de reforestation appelés à remédier au recul des essences forestières exigeantes en eau. De nombreux travaux attestent la dégradation des pistachiers dans les Dayas (climat aride au sens large) et la rattachent à l'action de l'homme. L'absence de données météorologiques complètes sur de longues périodes dans l'aire du pistachier pose cependant le problème de connaissances approfondies de la variabilité spatio-temporelle du climat de ces régions. En effet, les quelques stations météorologiques disponibles, le plus souvent récentes et installées en milieu urbain, ne traduisent pas d'une manière assez fiable ces changements.

Pour pallier ce déficit, nous avons utilisé la « mémoire » de l'arbre pour extraire les signaux enregistrés dans le bois à l'aide de la dendroécologie. Ce travail fait suite à une étude déjà réalisée sur la croissance radiale et la sensibilité au climat du pistachier de l'Atlas (Pistacia atlantica Desf.) en Algérie dans deux régions bioclimatiques (semi-aride au Centre algérien et aride à l'extrême Ouest) (Ifticene-Habani et Messaoudène, 2016). Notre objectif est l'analyse des relations cerne-climat chez le pistachier de l'Atlas afin de fournir une vision plus complète du déterminisme climatique de la croissance radiale, et mieux appréhender l'impact futur des changements environnementaux sur la croissance et la vitalité de l'espèce.

\section{Matériels et méthodes}

\section{Présentation de l'espèce et site d'étude}

Le pistachier de l'Atlas est, par sa dioécie et ses fleurs nues, une espèce particulière, relevant de la famille des anacardiacées et du genre Pistacia (Gaussen et al., 1982). Les fleurs mâles apparaissent avant les fleurs femelles, la pollinisation est anémophile, rarement entomophile, et coïncide avec la période des vents dominants durant le mois de mars. La reproduction sexuée aboutit le plus souvent à la production de fruits parthénocarpiques (Morsli, 1992). Monjauze (1968) note, en se référant à la disposition des semis naturels, que la dissémination des graines du pistachier de l'Atlas se fait par les oiseaux.

Zohary (1952) a divisé ce genre en quatre sections et 11 espèces selon la morphologie foliaire et a décrit Pistacia atlantica comme comprenant deux sous-espèces $-P$. kurdica (Zohary) et $P$. latifolia DC. Parfitt et Badenes (1997) notent l'existence de la sous-espèce atlantica Desf. qui pousse à l'état sauvage dans les régions arides et semi-arides de l'Algérie.

Au plan écologique, le pistachier de l'Atlas se développe sous une tranche pluviométrique allant de $250 \mathrm{~mm}$ à 1000 mm (Monjauze, 1968), voire moins de $100 \mathrm{~mm}$ au Hoggar. D’après Kaska (1994), il supporte aussi bien les températures basses $\left(-12^{\circ} \mathrm{C}\right)$ que les températures élevées $\left(49^{\circ} \mathrm{C}\right)$. En milieu humide, le pistachier de l'Atlas accompagne les essences forestières d'altitude. En revanche, il constitue des groupements préforestiers climaciques en bioclimat semi-aride (Monjauze, 1968 ; Achhal et al., 1980). Sa physiologie est très peu étudiée et, bien qu'ils pourraient apporter un éclairage sur ses adaptations au climat, ces travaux sont à l'état d'ébauche (Belhadj, 2007 ; Amara, 2014 ; Limane et al., 2014).

La présente étude a été réalisée à Djebel Meddad, au sein du Parc national de Theniet El Had qui fait partie de la région orientale de l'Ouarsenis (principal chaînon du Tell occidental) (Sari, 1977). Il s'agit d'une aire de 3425 ha protégée depuis 1983 où les traitements sylvicoles se limitent à des coupes sanitaires dans la cédraie en particulier. Le pâturage, toutefois, limite la régénération du pistachier de l'Atlas. Deux stations ont été choisies selon l'exposition du massif (ubac et adret) (figure 1).

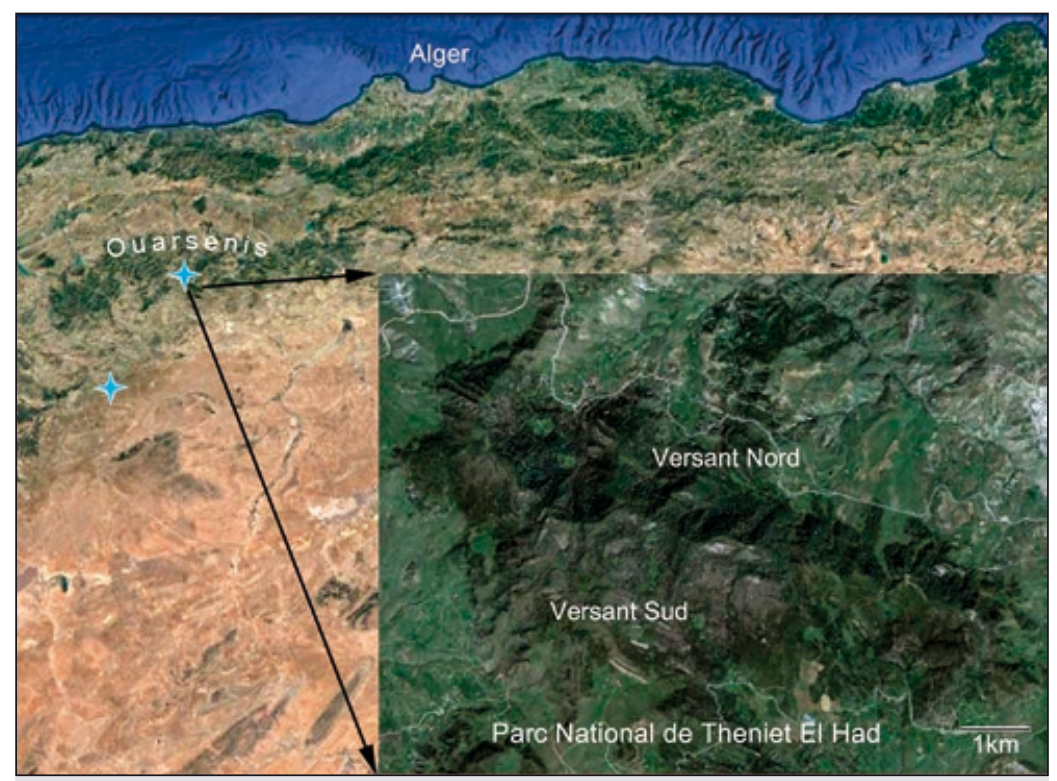

Figure 1.

Carte de situation géographique de la zone d'étude (source : Google Earth).

+ Station météorologique. 


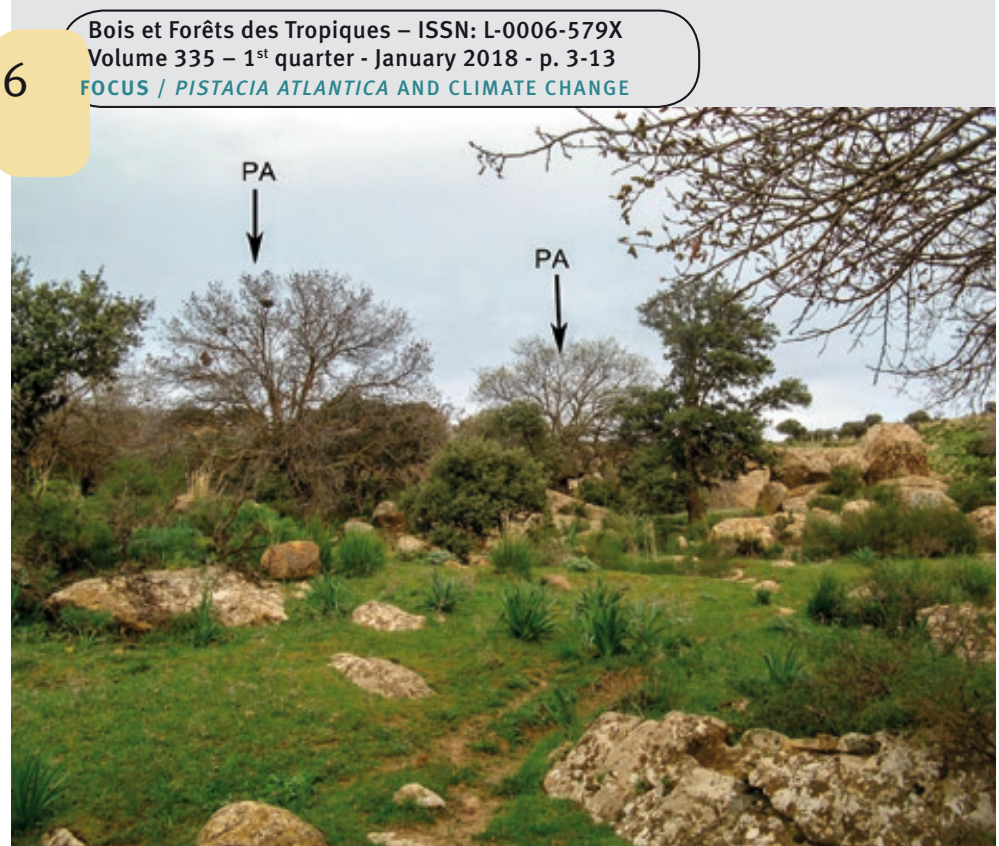

Photo 2.

Vue du versant sud de Djebel Meddad où le pistachier de l'Atlas (PA) accompagne le chêne vert (avril 2012). Photo F. Abdoun.

Sur le versant nord, le pistachier de l'Atlas cohabite avec plusieurs essences forestières, notamment le cèdre de l'Atlas et le chêne vert. Dans les stations les plus humides, il est en mélange avec le chêne vert et le chêne zéen. Sur le versant sud, l'espèce est mêlée au chêne vert, au chêneliège et au pin d'Alep (photos 1 et 2).

La station météorologique retenue pour cette étude est celle de Tiaret. Elle se situe à $60 \mathrm{~km}$ au sud-ouest et réunit des conditions bioclimatiques assez proches de celles de Theniet El Had (Stewart, 1969). Les données sont complètes pour les précipitations et les températures moyennes annuelles. Les données climatiques sont fournies par l'Office national de météorologie (ONM) pour la période 1983-2011. La seconde station météorologique retenue est celle de Theniet El Had, où ne sont disponibles que les précipitations pour la période 1952-2011. Ces données sont fournies par l'Agence nationale des ressources hydrauliques (ANRH). La figure 2 illustre le diagramme ombrothermique de la station météorologique de Tiaret.

Le tableau I résume les caractéristiques physiques des stations et leur situation géographique.

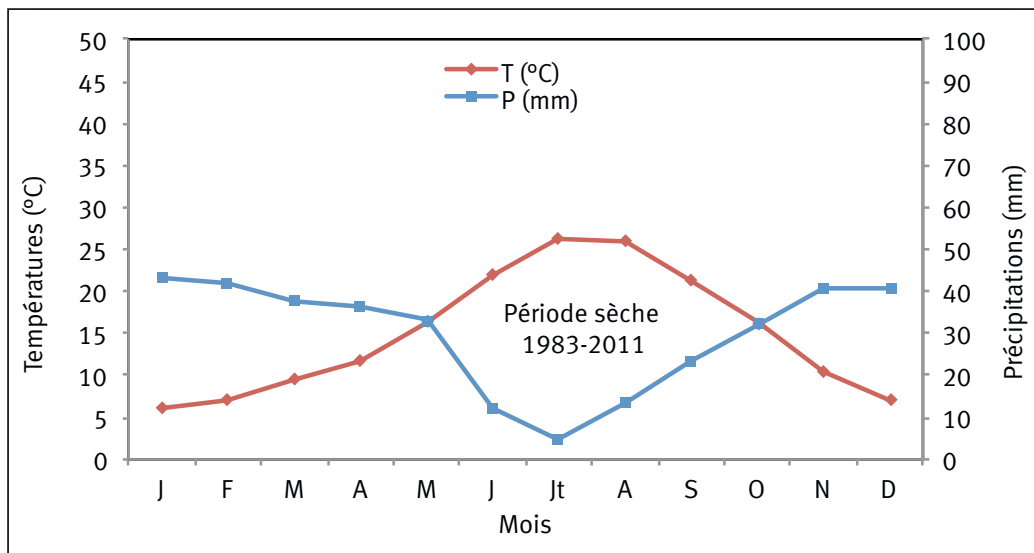

Figure 2.

Diagramme ombrothermique de Bagnouls et Gaussen de la station météorologique de Tiaret.

\section{Échantillonnage et traitement des données}

Seize arbres ont été sondés sur l'ubac et 29 arbres sur l'adret. Les individus sélectionnés répondent aux critères établis par Stokes et Smiley (1968). Pour chaque arbre, trois carottes ont été extraites à l'aide d'une tarière de Pressler, à une hauteur de tronc égale à 1,30 m. Les carottes ont été collées sur des réglettes de bois rainurées, puis poncées pour faciliter la lecture et les mesures des cernes. Les mesures des épaisseurs des cernes ont été réalisées à une précision de $1 / 1000 \mathrm{~mm}$ au laboratoire de la qualité du bois à la station régionale de l'Institut national de recherche forestière d'Azzazga (Tizi Ouzou). L'interdatation s'est avérée difficile étant donné la présence, dans certains échantillons, de zones à cernes très minces voire absents, à l'anatomie du bois à pores diffus, et à la présence de faux cernes ou cernes doubles dans le bois initial, surtout pour les échantillons du versant sud (photo 3). Ce phénomène a été observé pour la même espèce dans les régions arides et semi-arides (IfticeneHabani et Messaoudène, 2016). Ainsi, seulement 12 arbres sur $16(75 \%)$ ont été interdatés pour la population du Nord et 20 arbres sur 29 (69\%) pour la population du Sud.

Une autre caractéristique a été observée dans le bois du pistachier de l'Atlas dans la région d'étude par rapport aux régions aride et semi-aride; il s'agit de la couleur brun foncé à noire allant du centre jusqu'à l'écorce. Boudouaya et al. $(2015,2016)$ en ont déduit que cette couleur provenait d'une substance (résine) qui confère au bois du pistachier de l'Atlas une durabilité élevée. Cependant, avec l'âge, le bois de cœur se détériore par la pourriture qui entame le duramen et avance en flamme vers l'aubier (photos 4 et 5). De ce fait, la longueur des séries de cernes est limitée.

L'analyse de la réponse aux événements climatiques extrêmes par le calcul des années caractéristiques, appelées aussi pointer years (Schweingruber et al., 1990), a été réalisée à l'aide de l'application POINTER développée sous le logiciel libre R (R Development Core Team, 2011) par Mérian (2012a). POINTER permet de calculer les années caractéristiques à partir des épaisseurs brutes de cernes interdatés, selon la méthode développée par Becker (1989). L'année est positive en cas de croissance plus forte, et négative dans le cas contraire. Ensuite, les chronologies individuelles brutes ont été standardisées à l'aide de l'application DENDRO développée sous R par Mérian (2012a), selon le processus classique de la double pondération, afin d'éliminer les signaux non liés au climat (Cook, 1985). DENDRO permet aussi de calculer des fonctions de réponse et de corrélation (BCC) entre les indices de croissance et les régresseurs climatiques (Fritts, 1976 ; Cook et Kairiukstis, 1990 ; Guiot, 1991). Les variables climatiques utilisées couvrent la période de septembre de l'année précédente $t-1$ à août de l'année en cours $t$ (12 mois) pour les températures moyennes mensuelles et les cumuls de précipitations mensuels. La mise en forme et la standardisation des séries climatiques ont été réalisées par l'application CLIMAT (Mérian, 2012a) pour leur utilisation dans DENDRO. Divers paramètres statistiques ont été calculés afin de juger de la qualité et de la force du signal climatique contenu dans les cernes. Il s'agit : de la sensitivité 
Tableau I.

Caractéristiques physiques des stations.

\begin{tabular}{|c|c|c|}
\hline Caractéristiques & Versant nord & Versant sud \\
\hline Longitude & $1^{0} 57,605^{\prime} \mathrm{E}$ et $1^{\circ} 57,970^{\prime} \mathrm{E}$ & $1^{0} 59,579^{\prime} \mathrm{E}$ et $2^{\circ} 00,523^{\prime} \mathrm{E}$ \\
\hline Latitude & $35^{\circ} 52,391^{\prime} \mathrm{N}$ et $35^{\circ} 52,767^{\prime} \mathrm{N}$ & $35^{\circ} 49,611^{\prime} \mathrm{N}$ et $35^{\circ} 50,495^{\prime} \mathrm{N}$ \\
\hline Altitude (m) & 1245 à 1312 & 1081 à 1273 \\
\hline Exposition & Nord et Nord-Est & Sud \\
\hline Pente (\%) & 35 à 40 & 0 à 30 \\
\hline Élément floristique & Cèdre de l’Atlas, chêne vert et chêne zéen & $\begin{array}{l}\text { Chêne-liège, pin d’Alep, chêne vert } \\
\text { et genévrier de Phénicie }\end{array}$ \\
\hline Élément topographique & Haut et mi-versant & Mi- et bas versant \\
\hline Élément géologique & Sédiments oligocènes & Sédiments oligocènes \\
\hline Élément pédologique: & Sols peu évolués d'apport colluvial & Sols peu évolués d'apport colluvial \\
\hline Bioclimat & Subhumide frais & Subhumide frais \\
\hline $\begin{array}{l}\text { Hauteur totale moyenne } \\
\text { du peuplement }(\mathrm{m})\end{array}$ & 12 & 8 \\
\hline $\begin{array}{l}\text { Circonférence moyenne } \\
\text { du peuplement }(\mathrm{m})\end{array}$ & 3 & 2 \\
\hline $\begin{array}{l}\text { Période couverte par } \\
\text { chronologie moyenne }\end{array}$ & $1942-2011$ & $1923-2011$ \\
\hline
\end{tabular}

moyenne (SM) qui évalue la variabilité moyenne d’épaisseur entre deux cernes successifs et exprime l'importance des changements climatiques à court terme affectant ces derniers ; du coefficient d'autocorrélation (AC) d'ordre 1 qui exprime l'effet de la persistance lié aux conditions conduisant à la formation du cerne de l'année $t-1$ sur la mise en place du cerne de l'année suivante $t$ (Fritts, 1976) ; et de l'Expressed Population Signal (EPS) qui reflète la part de signal commune aux arbres échantillonnés et permet d'estimer la force du signal contenu dans les cernes étudiés (Wigley et al., 1984 ; Mérian et Lebourgeois, 2011).

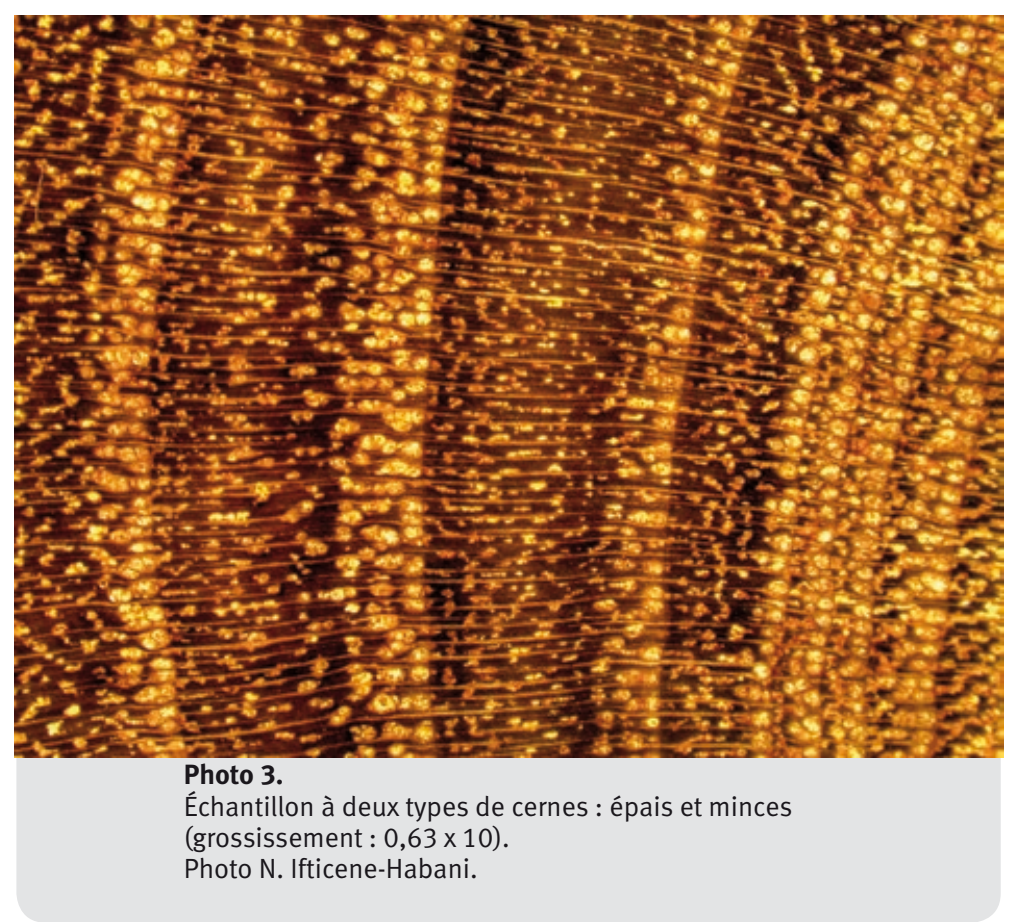

\section{Résultats}

\section{Variation de l'épaisseur brute des cernes}

Le tableau II rapporte les valeurs de l'épaisseur moyenne du cerne (EMC). Ces valeurs sont sensiblement supérieures dans le versant sud du massif à celles du versant nord, avec un maximum de 4,02 $\mathrm{mm}$. Les valeurs élevées des écarts-types et des coefficients de variation traduisent la variabilité temporelle à l'intérieur d'une même chronologie.

La sensitivité moyenne (sens1) calculée sur les chronologies de synthèses brutes présente des valeurs très proches dans les deux stations. Le résultat obtenu du coefficient d'autocorrélation d'ordre 1 est supérieur à 0,5 . Ces valeurs expriment le phénomène de «persistance » et indiquent que le cerne de l'année $t$ se trouve déjà prédéterminé en grande partie par la croissance et les conditions climatiques de l'année antérieure $t-1$.

\section{Années caractéristiques et climat}

Les années caractéristiques ont été calculées sur la période 1943-2011 (tableau III). Le nombre total d'années caractéristiques est de quatre pour la station nord et 17 pour la station sud. Le pistachier présente plus d'années caractéristiques négatives que positives (12 contre 9 ). Toutes les années caractéristiques correspondent à des conditions particulièrement sèches ou pluvieuses (action directe), à l'exception de l'année 2010 (action inverse). Les résultats obtenus pour les années caractéristiques 


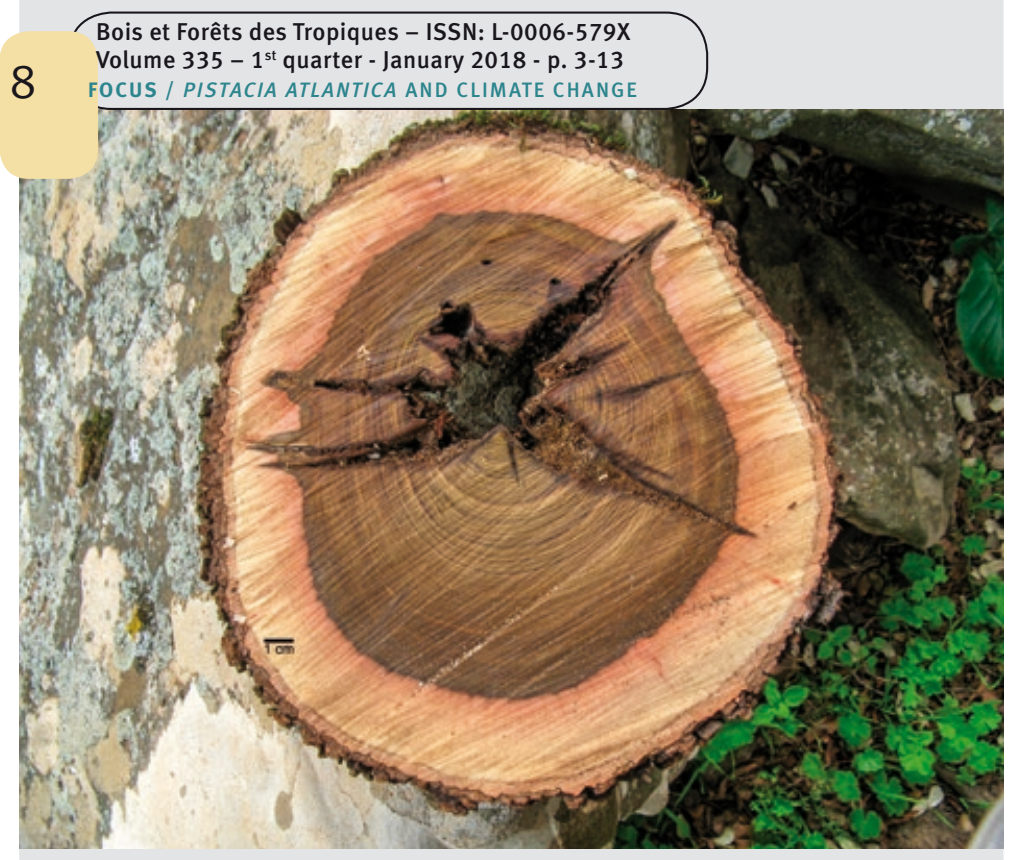

Photo 4.

Bois du pistachier de l'Atlas pourri (premier stade). Photo F. Abdoun.

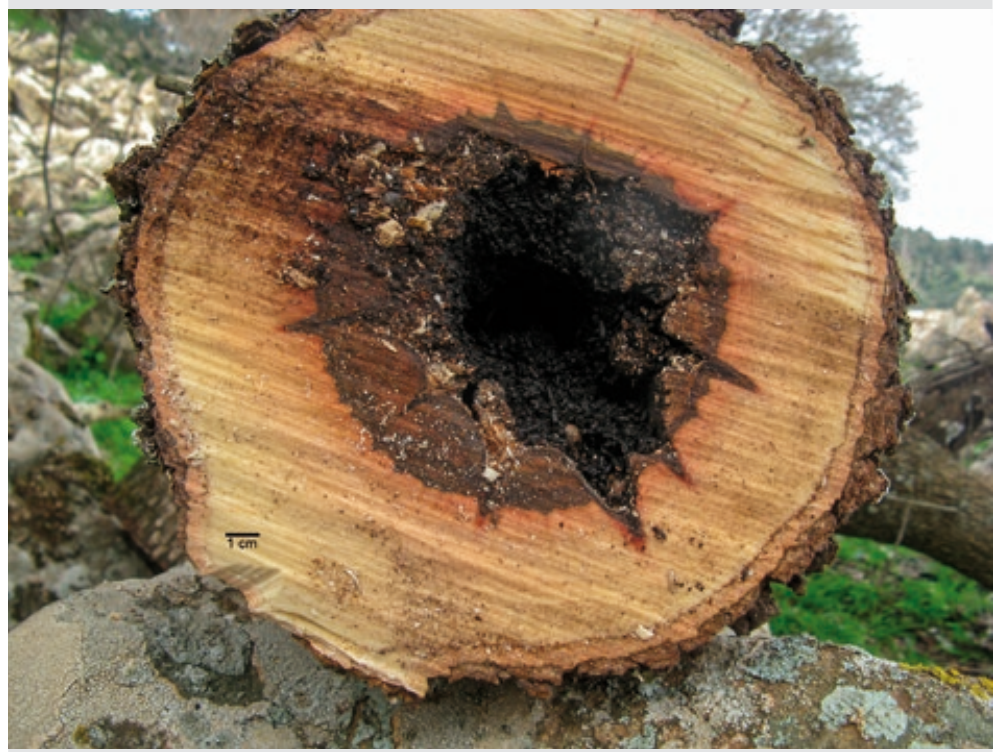

Photo 5.

Bois du pistachier de l'Atlas pourri (stade plus avancé). Photo F. Abdoun.

1945, 1946, 1948 et 1951 ne peuvent être expliqués du fait de l'absence de données de précipitations. Pour l'année 1952, une reprise de croissance est notée pour la station sud malgré des précipitations égales à la moyenne. Les cernes épais de 1955 et 1959 peuvent être dus aux précipitations supérieures à la moyenne enregistrée en 1953. Le déficit hydrique des années 1982 et 1994 ainsi que le froid des années 1984, 1991 et 1992 ne semblent pas affecter la croissance radiale de l'espèce. Au cours de l'année 2000, marquée par des conditions climatiques sévères, le pistachier de l'Atlas n'a pas enregistré une chute de croissance.
Tableau II.

Paramètres statistiques calculés

sur les séries brutes des stations.

\begin{tabular}{|c|c|c|}
\hline \multirow[t]{2}{*}{ Paramètres } & \multicolumn{2}{|c|}{ Station } \\
\hline & Versant nord & Versant sud \\
\hline EMC moy. (mm) & 1,54 & 1,88 \\
\hline EMC min. (mm) & 0,86 & 1,02 \\
\hline EMC max. (mm) & 2,56 & 4,02 \\
\hline ET & 0,22 & 0,21 \\
\hline $\mathrm{CV}$ & 22,52 & 21,16 \\
\hline sens1 moy. & 0,27 & 0,28 \\
\hline sens1 min. & 0,18 & 0,21 \\
\hline sens1 max. & 0,37 & 0,40 \\
\hline $\operatorname{ar1}$ & 0,59 & 0,58 \\
\hline \multicolumn{3}{|c|}{$\begin{array}{l}\text { EMC : épaisseur moyenne du cerne ; ET : écart-type ; } \\
\text { CV : coefficient de variation ; sens1 : sensitivité moyenne } \\
\text { (minimale et maximale), évalue la variabilité moyenne } \\
\text { d'épaisseur entre deux cernes successifs ; } \\
\text { ar1 : coefficient d'autocorrélation d'ordre } 1 .\end{array}$} \\
\hline
\end{tabular}

Afin de mieux comprendre le rôle des précipitations dans la croissance radiale du pistachier de l'Atlas, nous avons calculé la moyenne des précipitations saisonnières pour les années caractéristiques positives et négatives. Les résultats présentés dans le tableau III montrent que la distribution des pluies en fonction des saisons ne semble pas affecter la croissance de l'espèce. On remarque les mêmes régimes saisonniers pour les années caractéristiques négatives et les années caractéristiques positives.

\section{Standardisation des épaisseurs des cernes}

La standardisation vise à transformer les épaisseurs brutes en indices de dimension uniforme. Elle entraîne une uniformisation des variations de croissance indépendamment des différences d'âge et de croissance entre les arbres échantillonnés. Elle élimine les signaux non désirés, c'est-à-dire les composantes non stationnaires (variables au cours du temps), notamment les fluctuations dues à l'âge de l'arbre (Fritts, 1976 ; Cook et Kairiukstis, 1990).

Les sensitivités moyennes calculées sur les chronologies standardisées sont plus faibles que celles calculées sur les séries brutes (tableau IV). Contrairement aux sensitivités des séries brutes qui sont très proches dans les deux stations, la population du sud semble plus sensible au climat avec sens $1=0,16$, contre sens $1=0,12$ au versant nord. Le coefficient d'autocorrélation d'ordre 1 est plus élevé en versant nord $(0,66)$ qu'en versant sud $(0,54)$. Les valeurs d'EPS sont comprises entre 0,67 au nord et 0,77 au sud. Ces valeurs sont assez proches du seuil de 0,85 (Wigley et al., 1984), surtout pour la population du sud, et suggèrent que les arbres réagissent de façon homogène au sein de cette dernière. 
Tableau III.

Années caractéristiques et années exceptionnelles.

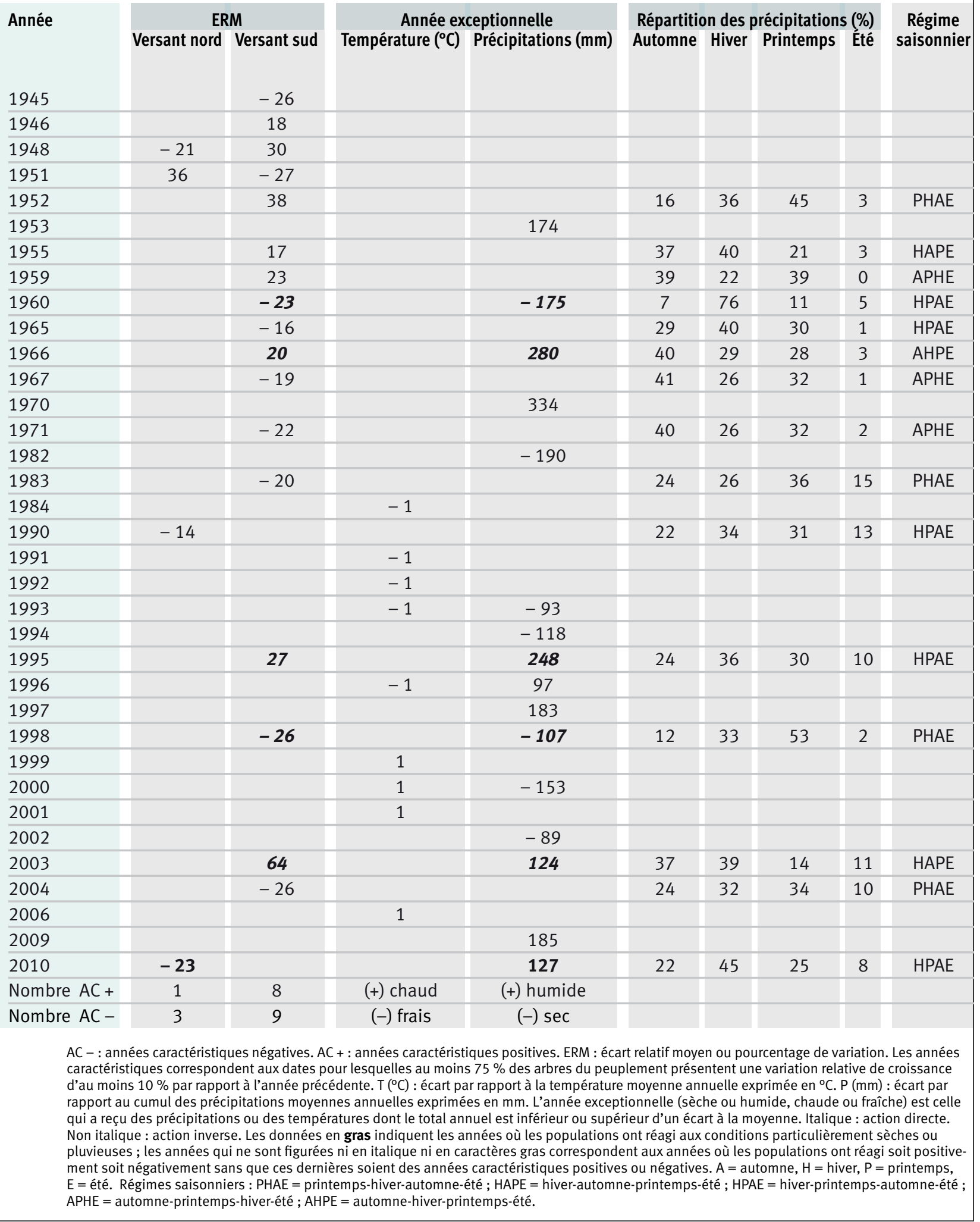




Tableau IV.
Paramètres dendrochronologiques
des chronologies standardisées.

\begin{tabular}{l|cc} 
Paramètres & \multicolumn{2}{c}{ Station } \\
& Versant nord & Versant sud \\
& & \\
IC moyen & 0,96 & 1,00 \\
\hline IC min. & 0,63 & 0,62 \\
\hline IC max. & 1,89 & 1,54 \\
\hline ET & 0,22 & 0,21 \\
\hline sens1 & 0,12 & 0,16 \\
\hline ar1 & 0,66 & 0,54 \\
\hline eps & 0,67 & 0,77
\end{tabular}

IC : indice de croissance ; EMC : épaisseur moyenne du cerne ; ET : écart-type ; sens 1 : sensitivité moyenne : évalue la variabilité moyenne d'épaisseur entre deux cernes successifs ; ar1 : coefficient d'autocorrélation d'ordre 1 ; eps : expressed population signal : estime la force du signal.
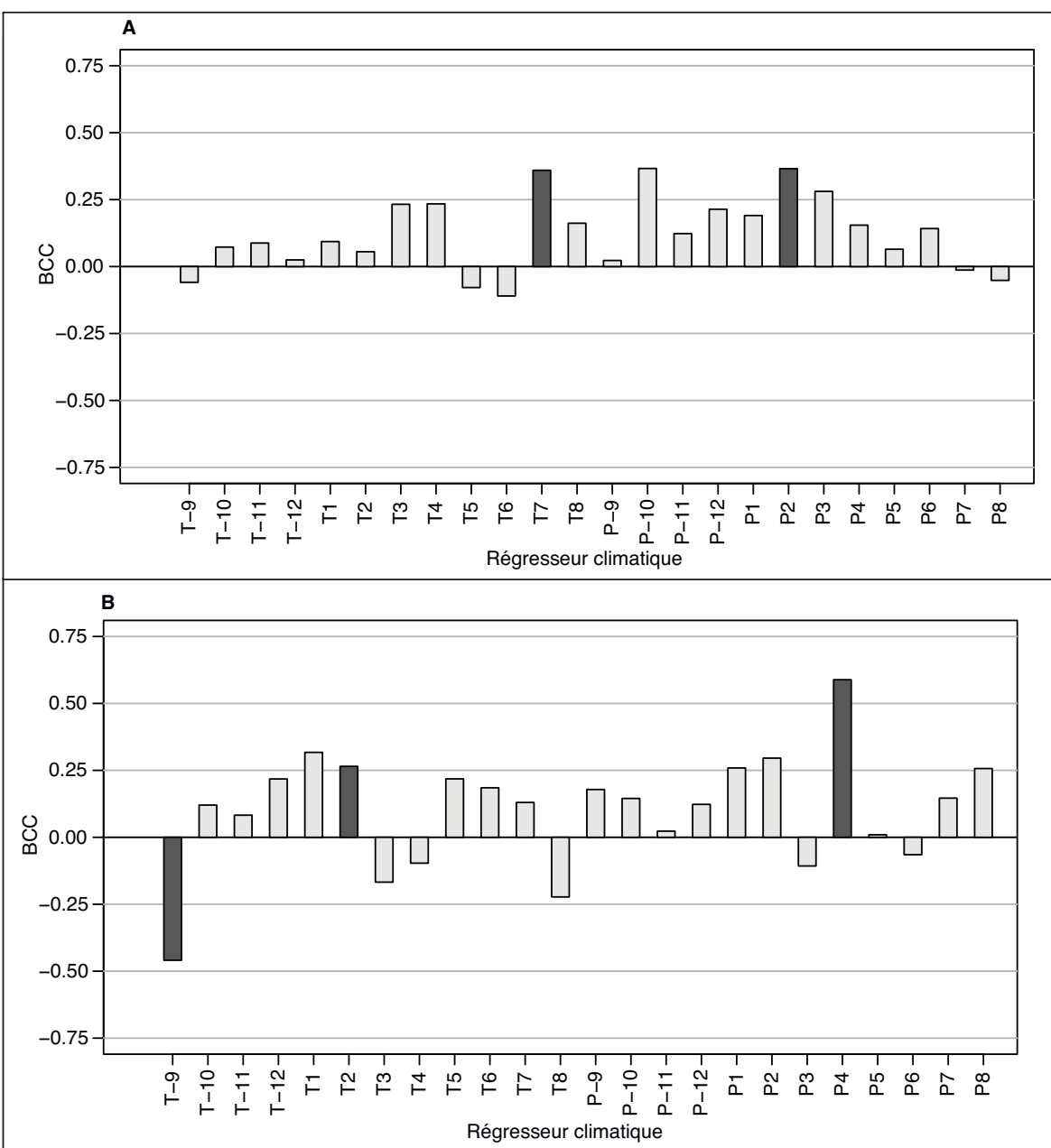

Figure 3.

Relations cerne-climat ( $\mathrm{A}$ : versant nord ; $B$ : versant sud). $B C C$ : coefficient de corrélation de type Bootstrap. $T$ : température. $P$ : précipitations (les mois sont indiqués par des chiffres : $T-9$ = septembre de l'année précédente ; T8 = août de l'année en cours). Les rectangles sombres traduisent un coefficient significatif au seuil de $95 \%$.
Les résultats présentés dans la figure 3 ( $A$ et $B$ ) montrent que, dans la même région, la réponse du pistachier de l'Atlas aux facteurs climatiques varie d'une station à une autre. Le nombre de mois au cours desquels les relations cerne-climat sont positivement significatives est de deux pour les deux versants. Au nord du massif, se dégage une relation positive significative avec les températures de juillet (T7) de l'année en cours. Au sud, la croissance radiale est négativement corrélée aux températures de septembre de l'année précédant la formation du cerne $(T-9)$. En revanche, les températures de février (T2) de l'année en cours jouent un rôle positif dans la croissance radiale de l'espèce. Une relation positive se distingue avec les précipitations de février (P2) de l'année en cours pour la population implantée au nord du massif. Au sud, les précipitations du mois d'avril de l'année en cours (P4) sont positivement corrélées à la croissance radiale.

\section{Discussion}

La variation observée de l'épaisseur brute des cernes pourrait être expliquée par la densité des peuplements et la différence des conditions du milieu entre les deux versants. Elle est supérieure à celle des stations de Djelfa et de Béchar (EMC en $\mathrm{mm}$ de 1,35 à 1,49 , et de 1,06 à 1,24) (Ifticene-Habani et Messaoudène, 2016). Selon Safar (1994), les particularités stationnelles induisent des variations différentielles de l'intensité de la réaction des arbres à des facteurs de l'environnement qui fluctuent d'une année à l'autre. Ceci peut être attribué au climat ou à la nature particulière du biotope. Le pistachier de l'Atlas étant un arbre de lumière, la population située sur le versant sud bénéficie de conditions clémentes garantissant une croissance radiale plus élevée, comme le soulignent Mérian et Lebourgeois (2011) et Lebourgeois et Mérian (2012). Les valeurs de la sensitivité moyenne (sens1) sont faibles par rapport à celles obtenues pour la même espèce dans les régions semiaride (Djelfa : 0,42< sens $1<0,49$ ) et aride (Béchar : 0,35<sens1<0,36) (Ifticene-Habani et Messaoudène, 2016). Dans la même région d'étude (massif de l'Ouarsenis), Sarmoum (2008) obtient pour le cèdre de l'Atlas des valeurs entre 0,19 et 0,43 et entre 0,17 et 0,33 pour le pin d'Alep (Sarmoum et al., 2016). Le pistachier de l'Atlas est plus sensible aux événements extrêmes dans les expositions chaudes que dans les expositions fraîches, plus particulièrement aux événements qui inhibent 
la croissance. En revanche, il peut bénéficier de l'effet différé de précipitations abondantes (année 1953) ou d'un effet de compensation de facteurs climatiques. Cherubini et al. (2003) rappellent la nécessité de prendre en compte les conditions stationnelles pour l'étude de la croissance des arbres en région méditerranéenne. Le pistachier de l'Atlas n'a pas enregistré une chute de croissance en 2000 bien que cette année soit marquée par des conditions climatiques sévères, contrairement à ce qui a été observé conjointement dans les hautes plaines steppiques et dans la région présaharienne (Ifticène-Habani et Messaoudène, 2016). Selon Mérian (2012b), les années caractéristiques sont très rarement communes à plus de deux régions, ce qui traduit une variabilité spatiale importante des réactions des espèces aux variations climatiques. Par ailleurs, les températures n'ont aucun effet notable sur la croissance radiale des pistachiers, contrairement aux stations arides où elles jouent un rôle complémentaire.

Les sensitivités moyennes calculées sur les chronologies standardisées sont inférieures à celles trouvées pour la même espèce dans les régions arides et semi-arides (Ifticene-Habani et Messaoudène, 2016). Ceci a été également observé sur le pin d’Alep (Safar, 1994). Cette différence peut être liée à la fois au biotope et au facteur génétique (Messaoudène, 1989). Les valeurs du coefficient d'autocorrélation d'ordre 1 expriment le phénomène de " persistance » comme pour les séries brutes (Fritts, 1976). Cet effet pourrait probablement atténuer les effets de la sécheresse de courte période et de sévérité modérée si les conditions antérieures sont favorables (Kherchouche et al., 2013).

La réponse moyenne au climat a révélé que le nombre de mois au cours desquels les relations cerne-climat sont significatives est moins élevé que chez les populations étudiées dans les régions arides et semi-arides (Ifticene-Habani et Messaoudène, 2016). La relation positivement significative avec les températures de juillet laisse penser qu'il y aurait soit une activité cambiale même pendant l'été, soit un épaississement des parois cellulaires. Au sud, la croissance radiale négativement corrélée aux températures de septembre peut être interprétée comme une restauration des réserves hydriques. En revanche, l'effet des températures de février peut être expliqué par le phénomène de rhizogenèse favorisé par des températures supérieures à la moyenne en hiver, qui améliorent la capacité d'absorption (Meddour, 1992). L'action directe des précipitations de février au versant nord, et celle d'avril au versant sud, sont liées à la phase optimale de l'activité cambiale. Durant cette période, les précipitations jouent un rôle majeur dans la construction du cerne. Les arbres réagissent positivement aux précipitations de cette période dans la mesure où ces quantités d'eau, nettement inférieures à celles des périodes précédentes, peuvent s'intégrer à la réserve hydrique des sols permettant effectivement ce stockage (Messaoudène, 1989). Lebourgeois (2006) note que le déficit hydrique du sol est le facteur le plus important pour expliquer les variations de croissance. Présent dans les régions arides et semi-arides, le pistachier de l'Atlas manifeste des traits adaptatifs en modulant sa croissance en fonction des précipitations. Cette capacité traduit une plasticité élevée face aux variations climatiques (Ifticene-Habani et Messaoudène, 2016). Les résultats montrent que, sous bioclimat subhumide, le pistachier présente des relations avec les précipitations moins fortes que sous bioclimat aride ou semi-aride (Ifticene-Habani et Messaoudène, 2016).

À Djebel Meddad, le pistachier est dominé par le cèdre de l'Atlas et le chêne zéen sur le versant nord. Il se rencontre en mélange de moindre densité avec les chênes et le pin d'Alep sur le versant sud. Cette variation de son statut social module fortement sa réponse au climat (Badeau et al., 1995 ; Mérian et Lebourgeois, 2011 ; Lebourgeois et al., 2014). Pour le hêtre et le sapin pectiné, les arbres dominants sont plus sensibles au climat (Lebourgeois et al., 2014). Selon Picard et al. (1999), cette différence de comportement peut s'expliquer par une meilleure exploration racinaire des arbres dominants ainsi que par une utilisation plus efficace de l'eau, de la lumière et de l'espace. Ainsi, plus la position hiérarchique est favorable, plus l'amplitude des variations interannuelles (notamment durant les crises) est élevée. Bien que les arbres échantillonnés dans le versant sud soient tous de statut dominant, dans le versant nord, la différenciation sociale au sein du peuplement n'a pu être établie. Notre étude révèle que le pistachier de l'Atlas se développe aisément sur les deux versants dont les biotopes respectifs modulent l'influence des facteurs climatiques.

\section{Conclusion}

L'objectif poursuivi par cette étude était d'analyser des relations cerne-climat du pistachier de l'Atlas dans la région subhumide de Theniet El Had (Algérie). Les résultats montrent qu'il est possible de distinguer une largeur du cerne sensiblement supérieure sur le versant sud par rapport au versant nord. La variabilité interannuelle de la croissance montre la sensibilité au climat du pistachier de l'Atlas. La relation entre les années caractéristiques et les années exceptionnelles révèle, dans la majorité des cas, des années caractéristiques de croissance qui correspondent à des conditions particulièrement sèches ou humides. Les précipitations annuelles sont à l'origine de cette relation directe. Les fonctions de réponse montrent le rôle important des précipitations, mais moindre que sous bioclimat aride et semi-aride. Par contre, les températures n'exercent qu'un effet minime. L'analyse des relations cerne-climat montre la modulation de ce dernier par le biotope (topographie, sol, statut social des arbres).

Cette étude a certes été réalisée dans une région bien limitée au parc des cèdres de l'Ouarsenis, selon un échantillon réduit. Pour autant, ces résultats s'ajoutent à ceux relatifs aux bioclimats arides, et pourraient être complétés par la population sous climat saharien.

\section{Hommage}

Nous tenons à rendre hommage à l'initiateur de ce travail feu Mahand Messaoudène, directeur de recherche à l'Institut national de recherche forestière, station régionale d'Azazga, Tizi Ouzou, en Algérie. 


\section{Remerciements}

Nous remercions Mohamed Said Guettouche, directeur du Laboratoire de géographie et aménagement du territoire (USTHB), pour son aide dans l'application du programme R. Nous remercions également la direction du Parc national et la circonscription des forêts de Theniet El Had pour avoir facilité le travail de terrain.

\section{Références bibliographiques}

Achhal A., Akabli O., Barbero M., M’Hirit O., Peyre C., Quézel P., Rivas Martinez S., 1980. À propos de la valeur bioclimatique et dynamique de quelques essences forestières au Maroc. Ecologia Mediterranea, 5 : 211-249.

Amara M., 2014. Contribution à l'étude des groupements à Pistacia atlantica subsp. atlantica dans le Nord-Ouest algérien. Thèse de doctorat, Université Abou Bekr Belkaid, Tlemcen, Algérie, 214 p.

Badeau V., Dupouey J.-L., Becker M., Picard J.-F., 1995. Longterm growth trends of Fagus sylvatica L. in northeastern France. A comparison between high and low density stands. International Journal of Ecology, 16 (5): 571-583.

Becker M., 1989. The role of climate on present and past vitality of silver fir forests in the Vosges mountains of northeastern France. Canadian Journal of Forest Research, 19 (9): 1110-1117.

Belhadj S., 2007. Étude éco-botanique de Pistacia atlantica Desf. (Anacardiaceae) en Algérie, préalable à la conservation des ressources génétiques de l'espèce et sa valorisation. Thèse de doctorat, Université Mouloud Mammeri, Tizi Ouzou, Algérie, 183 p.

Benabadji N., Bouazza M., 2000. Quelques modifications climatiques intervenues dans le sud-ouest de l'Oranie (Algérie occidentale). Revue des Énergies Renouvelables, $3: 117-125$.

Boudouaya M., Benhassaini H., Bendimered-Mouri F. Z., Mothe F., Fournier M., 2015. Évaluation de la durabilité naturelle du bois de Pistacia atlantica Desf. du Nord de l'Algérie. Bois et Forêts des Tropiques, 325 (3) : 49-58. http://bft. cirad.fr/cd/BFT 325 49-58.pdf

Boudouaya M., Benhassaini H., Bendimered-Mouri F. Z., Mothe F., Fournier M., 2016. Évaluation de la variabilité de la couleur du bois de Pistacia atlantica Desf. du Nord de l'Algérie. Bois et Forêts des Tropiques, 330 (4) : 23-35. http:// bft.cirad.fr/cd/BFT_330_23-35.pdf

Bréda N., Granier A., Aussenac G., 2004. La sécheresse de 2003 dans le contexte climatique des 54 dernières années : analyse écophysiologique et influence sur les arbres forestiers. Revue Forestière Française, 56 (2) : 109-131.

Cherubini P., Gartner B. L., Tognetti R., Bräker O. U., Schoch W., Innes J. L., 2003. Identification, measurement and interpretation of tree rings in woody species from mediterranean climates. Biological Reviews of the Cambridge Philosophical Society, 78: 119-148.
Cook E. R., 1985. A time series analysis approach to tree ring standardization. Tucson, USA, University of Arizona, Faculty of the School of Renewable Natural Ressources, 171 p.

Cook E. R., Kairiukstis L. A. E., 1990. Methods of dendrochronology: Applications in the environmental sciences. Dordrecht, Netherlands, Kluwer Academic Publishers, 408 p.

Fritts H. C., 1976. Tree rings and climate. London, New York, San Francisco, Academic Press, 567 p.

Gaussen H., Leroy J.-F., Ozenda P., 1982. Précis de botanique. 2. Végétaux supérieurs. $2^{\mathrm{e}}$ édition. Paris, France, Masson, 579 p.

Guiot J., 1991. Methods and programs of statistics for paleoclimatology and paleoecology. In: Guiot J., Labeyrie L. (éds). Quantification des changements climatiques : Méthodes et programmes. Monographie 1, INSU, PNEDC, 253 p.

Hassini N., Abderrahmani B., Dobbi A., 2015. Changement climatique et phénomènes extrêmes : action symbiotique du climat et du milieu naturel dans l'occurrence de la sécheresse en Algérie. In : XXVIII Colloque de l'Association internationale de climatologie, Liège, Belgique, 503-508.

Hirche A., Boughani A., Salamani M., 2007. Évolution de la pluviosité annuelle dans quelques stations arides algériennes. Sécheresse, 18 (04) : 314-320.

Ifticene-Habani N., Messaoudène M., 2016. Croissance radiale et sensibilité au climat du pistachier de l'Atlas, Pistacia atlantica Desf., en Algérie. Bois et Forêts des Tropiques, 329 (3) : 3-15. http://bft.cirad.fr/cd/BFT 329 3-15.pdf

IPCC, 2013. Climate Change 2013: The Physical Science Basis. Contribution of Working Group I to the Fifth Assessment Report of the Intergovernmental Panel on Climate Change. Cambridge, UK, New York, USA, Cambridge University Press, $27 \mathrm{p}$.

Kaska N., 1994. The Pistachio in its traditional growing areas. CIHEAM-FAO, cours approfondi, production et économie des fruits secs, $17 \mathrm{p}$.

Kherchouche D., Kalla M., Gutierrez E., Briki A., Hamchi A., 2013. La sécheresse et le dépérissement du cèdre de l'Atlas (Cedrus atlantica Manetti) dans le massif du Belezma (Algérie). Sécheresse, 24 : 129-37.

Landmann G., Bréda N., Houllier F., Dreyer E., Luc-Flot J., 2003. Sécheresse et canicule de l'été 2003 : quelles conséquences pour les forêts françaises. Revue Forestière Française, 55 (4) : 298-308.

Lebourgeois F., 1999. Analyse du bilan hydrique et de la croissance des arbres dans le RENECOFOR. Rapport scientifique final. Union européenne, DG VI, projet $n^{\circ}$ 9760FR0030, INRA, $72 \mathrm{p}$.

Lebourgeois F., 2006. Sensibilité au climat des Chênes sessile et pédonculé dans le réseau RENECOFOR. Comparaison avec les Hêtraies. Revue Forestière Française, 68 (1) : 29-44. Lebourgeois F., Mérian P., 2012. Principes et méthodes de la dendrochronologie. AgroParisTech, ENGREF, 85 p. https:// www.researchgate.net/profile/Francois Lebourgeois/publication/235989567 Principes et methodes de de la dendrochronologie/links/02e7e51543aaf00764000000/ Principes-et-methodes-de-de-la-dendrochronologie.pdf 
Lebourgeois F., Eberlé P., Mérian P., Seynave I., 2014. Social status-mediated tree-ring responses to climate of Abies alba and Fagus sylvatica shift in importance with increasing stand basal area. Forest Ecology and Management, 328: 209-218.

Limane A., Smail-Saadoun N., Gradziel T., 2014. Root architecture of Atlas pistachio in relation to underlying soil properties under arid conditions. African Journal of Agricultural Research, 9 (6): 622-625.

Meddour H., 1992. Contribution à l'étude dendrochronologique de deux provenances de pin d'Alep (Pinus halepensis) en Algérie. Cas d'une station littorale et d'une autre continentale. Thèse de magistère, INA, Alger, Algérie, 178 p.

Mérian P., 2012a. POINTER et DENDRO : deux applications sous $R$ pour l'analyse de la réponse des arbres au climat par approche dendroécologique. Revue Forestière Française, 64 (6) : 789- 798.

Mérian P., 2012b. Variations spatio-temporelles de la réponse au climat des essences forestières tempérées : quantification du phénomène par approche dendroécologique et influence de la stratégie d'échantillonnage. Thèse de doctorat, Institut des sciences et industries du vivant et de l'environnement, AgroParisTech, France, 451 p. https://pastel.archives-ouvertes.fr/pastel-00764526

Mérian P., Lebourgeois F., 2011. Consequences of decreasing the number of cored trees per plot on chronology statistics and climate-growth relationships: a multispecies analysis in a temperate climate. Canadian Journal of Forest Research, 41 (12): 2413-2422.

Messaoudène M., 1989. Dendroécologie et productivité de Quercus afares Pomel et Quercus canariensis Willd. dans les massifs forestiers de l'Akfadou et de Beni Ghorbi en Algérie. Thèse de doctorat, Université d'Aix-Marseille III, France, 123 p. Monjauze A., 1968. Répartition et écologie de Pistacia atlantica Desf. en Algérie. Bulletin de la Société d'Histoire Naturelle d'Afrique du Nord, $56: 1-127$.

Morsli A., 1992. Analyse de la floraison et de la structure sexuelle d'un peuplement de Pistacia atlantica Desf. dans une Daya de la région de Messaad. Mémoire ingénieur d’État, INA, Algérie, 60 p.

Parfitt D. E., Badenes M. L., 1997. Phylogeny of the Genus Pistacia as determined from analysis of the chloroplast genome. Proceedings of the National Academy of Sciences, USA, 94: 7987-7992.

Picard J. F., Becker M., Nys C., Dupouey J.-L., 1999. Évolution à moyen terme de la croissance radiale de l'épicéa et du hêtre en relation avec la fertilisation/amendement : analyse dendroécologique. Revue Forestière Française, 51 (2) : 197-218.

R Development Core Team, 2011. R: A language and environment for statistical computing. Vienna, Austria, R Foundation for Statistical Computing. http://www.r-project.org

Safar W., 1994. Contribution à l'étude dendroécologique du pin d'Alep (Pinus halepensis Mill.) dans une région semi-aride d'Algérie : l'Atlas Saharien (Ouled Naïl - Aurès - Hodna). Thèse de doctorat, Université d'Aix-Marseille III, France, $215 \mathrm{p}$.
Sala O. E., Chapin III S. F., Armesto J. J., Berlow E., Bloomfield J., Dirzo R. et al., 2000. Global biodiversity scenarios for the year 2100. Science, 287: 1770-1774.

Sari Dj., 1977. L'Homme et l'érosion dans l'Ouarsenis. Alger, Algérie, Éditions SNED, 628 p.

Sarmoum M., 2008. Impact du climat sur le dépérissement du cèdre de l'Atlas (Cedrus atlantica Manetti). Diagnostic dendroécologique et climatique de la cédraie de Theniet $\mathrm{El}$ Had (Wilaya de Tissemsilt). Thèse de magister, USTHB, Algérie, $98 \mathrm{p}$.

Sarmoum M., Guibal F., Abdoun F., 2016. Effet des facteurs stationnels sur la croissance radiale et la réponse du pin d'Alep au climat dans le massif de l'Ouarsenis, Algérie. Bois et Forêts des Tropiques, 329 (3) : 17-27. http://bft.cirad.fr/ cd/BFT 329 17-27.pdf

Schweingruber F. H., Eckstein D., Serre-Bachet F., Brakero U., 1990. Identification, presentation and interpretation of event years and pointer years in dendrochronology. Dendrochronologia, $8: 9-38$.

Stewart P. H., 1969. Quotient pluviothermique et dégradation de la biosphère. Quelques réflexions. Bulletin de la Société d'Histoire Naturelle d'Afrique du Nord, 59 : 23-37.

Stokes M. A., Smiley T. L., 1968. An introduction to tree ring dating. Chicago, USA, The University of Chicago Press, 73 p.

Wigley T. M., Briffa K. R., Jones P. D., 1984. On the average value of correlated time series, with applications in dendroclimatology and hydrometeorology. Journal of Climate and Applied Meteorology, 23: 201-213.

Zohary M., 1952. A monographical study of the genus Pistacia. Palestine Journal of Botany, 5: 187-228.

Bois et Forêts des Tropiques - Revue scientifique du Cirad
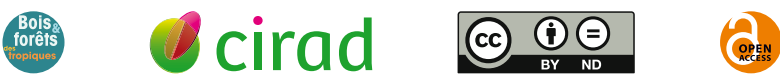

Cirad - Campus international de Baillarguet, 34398 Montpellier Cedex 5, France - Contact : bft@cirad.fr - ISSN : L-0006-579X 